\title{
Satisfacción con el empleo de extensionista rural: un estudio cualitativo en Caldas, Colombia ${ }^{1}$
}

\author{
Marlon Javier Méndez Sastoque ${ }^{2}$ \\ http://dx.doi.org/10.1590/0034-737X201562030003
}

\section{RESUMEN}

Como aporte al posicionamiento de la extensión rural como objeto multidimensional de estudio, se analizan las valoraciones realizadas por extensionistas rurales acerca de situaciones inherentes a su experiencia cotidiana de trabajo, seguido de la identificación de factores de satisfacción/insatisfacción laboral. En consonancia con la esencia cualitativa del estudio, la información se generó a partir de entrevistas semiestructuradas a 23 extensionistas rurales adscritos las Unidades Municipales de Asistencia Técnica (UMATA) de cinco municipios del departamento de Caldas, Colombia. El análisis de discurso permitió identificar ocho factores: a) gusto por la profesión ejercida, b) posibilidades de proyección social, c) alcance y visualización de logros, d) reconocimiento público, e) posibilidades de ascenso, f) posibilidades de aprendizaje continuo, g) autonomía, y h) estabilidad laboral. Se concluye que desconocer el estado emocional de los extensionistas hacia el trabajo produce el riesgo de crear o conservar escenarios que limiten el compromiso con la labor social realizada y el mantenimiento de la vocación extensionista.

Palabras clave: extensión rural, servicios de extensión agrícola, desarrollo rural.

\section{ABSTRACT}

\section{Job satisfaction among rural extension agents: a qualitative research in Caldas, Colombia}

As a contribution to the positioning of the rural extension as multidimensional object of study, we analyzed the assessments made by rural extension agents about situations inherent to their everyday experience of work, followed by the identification of factors of job satisfaction and dissatisfaction. Consistent with the qualitative nature of the study, the information was generated from semi-structured interviews with 23 rural extension agents affiliated to the Agency of Agricultural Technical Assistance (UMATA) in five municipalities of Caldas State, Colombia. The analysis identified eight factors: a) love for the profession, b) possibilities of social projection, c) scope and display of achievements, d) public recognition, e) promotion opportunities, f) possibilities of continuous learning, g) autonomy, h) labor stability. We concluded that ignoring the emotional condition of the extension agents towards the job produces the risk of creating or preserving scenes that limit the commitment with the social work practice realized and the preservation of the professional vocation.

Key words: rural extension; agricultural extension service; rural development.

\footnotetext{
Enviado el 31/10/2013 y aprobado el 15/04/2015.

${ }^{1}$ El artículo deriva del acompañamiento realizado por el autor a las Unidades Municipales de Asistencia Técnica Agropecuaria del departamento de Caldas, Colombia, para el fortalecimiento de la capacidad institucional.

22 Departamento de Desarrollo Rural y Recursos Naturales, Universidad de Caldas, Manizales, Caldas, Colombia. marlon.mendez@ucaldas.edu.co
} 


\section{INTRODUCCIÓN}

Quienes desde diferentes perspectivas estamos involucrados con el mundo rural, con frecuencia escuchamos o somos participes de conversaciones en la que asistentes técnicos o extensionistas rurales incluyen en sus discursos insinuaciones o comentarios directos sobre la forma como ellos mismos valoran sus empleos y oficio. Se trata de diálogos informales en los que, además de aludirse a las faenas diarias, se esbozan apuntes sobre la calidad de vida laboral.

Más que observaciones sobre la extensión rural en sí -a grandes rasgos asumida como práctica coadyuvante en los procesos destinados a generar condiciones ascendentes de bienestar entre los miembros de las comunidades rurales-, en estos diálogos salen a relucir juicios de conformidad e inconformidad con algunas situaciones inherentes a la experiencia diaria de trabajo, bien sea causantes de entusiasmo o intranquilidad. Sobre esta mirada, en un contexto social que asigna a los extensionistas el importante papel de impulsores directos o de base del desarrollo rural local y regional, surge una inquietud intelectual y pragmática: en su rol de trabajadores rurales (trabajadores al servicio del Estado que ejercen su oficio en el entorno rural y para el desarrollo rural), ¿se sienten satisfechos con su situación laboral? Llamar la atención sobre esto último, más sobre todo incitar el diálogo institucional y académico al respecto, es una de las razones motivadoras de la investigación.

Académicamente, dar respuesta a esta inquietud implica asumir, más que la extensión rural en sí, a los extensionistas y su condición de vida como objeto específico de estudio, acción poco evidente en los estudios especializados, en donde los extensionistas como tal figuran como los grandes ausentes. Por lo menos en el contexto latinoamericano, se ha dicho más acerca del perfil profesional, las políticas y los modelos de extensión agraria (Méndez 2006; Rodríguez et al., 2009; López y Castro, 2010; Rossi, 2011), que sobre el oficio de extensionista y su condición de trabajadores rurales. Sobre esta premisa, otro de los motivos que originó el estudio fue la intención de darles visibilidad y voz, permitiéndoles manifestar de qué manera viven, sienten y estiman sus condiciones de trabajo, esta vez en términos de satisfacción laboral.

Según Landy y Conte (2005), la satisfacción laboral es el estado emocional que resulta de la valoración del trabajo o de la experiencia laboral. De acuerdo con Spector (2012), es una variable de actitud que refleja las percepciones de las personas respecto a sus empleos en general, como bien en atención a aspectos específicos. Hontagas y Peiró (2002), asumiendo una postura multidimensional, la definen como una actitud genérica resultante de muchas actitudes específicas relacionadas con diversos aspectos del trabajo. Como es visible, se trata de definiciones que enfatizan la satisfacción laboral como actitud, concepto que es necesario aclarar y limitar.

Siguiendo lo expuesto por Sheth, Mittal y Newman (2001), las actitudes corresponden a las evaluaciones que las personas realizan de otras personas, eventos, objetos o cualquier otro elemento, en las que la calidad y la deseabilidad ocupan un papel primordial. Para Robbins y Coulter (2005), las actitudes son afirmaciones evaluativas, favorables o desfavorables en relación con eventos, objetos o personas, que reflejan cómo un individuo se siente en relación con alguna cosa. Similarmente, para Gamero (2005), representan una predisposición a responder de manera apacible o desaplacible a cierta situación vivida.

Para efectos del estudio, combinando lo expuesto por los autores citados, la satisfacción laboral es definida como la valoración evaluativa (emocional, cognitiva y afectiva) realizada por los extensionistas rurales en relación con su empleo en general y algunas situaciones específicas.

En este contexto, como aporte al posicionamiento de la extensión rural como objeto multidimensional de estudio, el objetivo del artículo es presentar, desde una perspectiva analítico-descriptiva, las valoraciones realizadas por los extensionistas rurales sobre diferentes circunstancias asociadas a su condición de trabajo, siendo esta la vía para el reconocimiento reflexivo de factores de satisfacción/insatisfacción laboral.

Lo propuesto se logró a partir de diálogos a profundidad con extensionistas rurales vinculados al sistema público de extensión, específicamente adscritos a las Unidades Municipales de Asistencia Técnica (UMATA) de cinco municipios del departamento de Caldas, Colombia, jurisdicción localizada en el centro occidente de la región andina nacional.

En cuanto al sitio de estudio, se trata de áreas rurales mayoritariamente dedicadas a la producción de café, en donde el cultivo de papa, cacao, caña panelera, tomate, cebolla, naranja y limón ocupa una mínima parte del espacio cultivado, por lo que el cultivo del café es el principal referente. Caldas cuenta con 40.123 caficultores, distribuidos en 52.075 predios, que en total suman 88.971 hectáreas sembradas en café. Del total de productores, $61,6 \%$ son minifundistas, caracterizados por vivir en el predio, tener menos de 1,5 hectáreas sembradas y un nivel educativo inferior a los cuatro años de escolaridad, siendo este el principal grupo poblacional al que las UMATA prestan sus servicios. Se trata de actores hoy empobrecidos y socioeconómicamente vulnerables, principalmente 
debido a la caída de las clausulas internacionales del café, la devaluación del dólar frente al peso colombiano, la presencia de nuevos problemas fitosanitarios y el deterioro de la institucionalidad para-estatal construida alrededor del cultivo (Nates y Vázquez 2009; Barón, 2010; Cano et al., 2012), sujetos en situación de desventaja, con quienes los extensionistas locales interactúan a diario.

En relación con los alcances de la investigación, vale decir que el estudio, por encima de pretender proporcionar claves factibles de ser utilizadas por los llamados ingenieros sociales para mejorar los sistemas de gestión de recursos organizacionales y humanos, busca proporcionar al público relacionado con la extensión y el desarrollo rural elementos para comprender la forma en que los extensionistas rurales viven, sienten, valoran y perciben su experiencia laboral hoy. En este contexto, se espera que lo hallado resulte útil, instigador o inspirador para quienes toman decisiones prácticas, definen políticas o ejecutan tareas referidas al desarrollo rural.

\section{MATERIALES Y MÉTODOS}

Como vía de aproximación a la realidad laboral de los extensionistas, atendiendo al carácter cualitativo del estudio, se optó por el método exploratorio descriptivo (Hesse-Biber y Leavy, 2010) en general, y la entrevista a profundidad, semiestructurada, en particular (Kvale, 1983; Kvale y Brinkmann, 2009). Con esta elección se consiguió explorar las percepciones genéricas de los extensionistas en relación con su experiencia laboral, así como captar múltiples detalles y matices sobre la forma como ellos actualmente la evalúan y valoran. Para ello se acudió al análisis minucioso de sus propias narrativas, identificando variables descriptivas recurrentes de satisfacción e insatisfacción con el trabajo.

Las entrevistas permitieron iniciar un diálogo fluido, espontáneo y profundo sobre la práctica laboral específica, con un estimuló consciente, mediante preguntas abiertas sobre los siguientes ejes de interés (Tabla 1).

Las conversaciones iniciaron con un primer estimulo verbal. Se preguntó: ¿usted se siente a gusto con su trabajo? Se prosiguió, acogiendo las recomendaciones dadas por Jodelet (2006), formulando preguntas más concretas relacionadas con la vida laboral, para posteriormente pasar a preguntas que incluyeran reflexiones más profundas, emociones y juicios de valor sobre esta misma experiencia.

En total se hicieron 23 entrevistas, entre noviembre de 2012 y marzo de 2013. El lugar y el momento elegido para realizarlas fueron negociados con los entrevistados, buscando contar con condiciones propicias de privacidad y tranquilidad. Dado lo comprometedor del tema, sobre todo cuando implica "hablar de lo negativo del trabajo (Entrevistado 16)", se evitó efectuarlas en las instalaciones de las UMATA, y en presencia de otras personas que pudieran distraer o inhibir a los entrevistados. Esto permitió lograr un mayor grado de intimidad y comodidad, favoreciendo el diálogo a profundidad y el acceso a información. En reconocimiento de lo expuesto, apelando a los principios de privacidad y confidencialidad, tanto los nombres de los entrevistados como de los municipios en los que prestan sus servicios son omitidos a lo largo del documento.

El tamaño de la muestra se definió usando la técnica de saturación (Morse, 1995). Se dejó de entrevistar e integrar nuevos casos al detectar que el contenido de las entrevistas se hacía repetitivo, por lo que entrevistar a una mayor cantidad de sujetos poco sumaba a los datos ya obtenido.

Una vez transcritas las entrevistas, los pasos seguidos para el tratamiento y análisis de datos fueron, en resumen, los siguientes: a) lectura repetitiva de los textos, b) selección inicial de situaciones recurrentes asumidas como variables descriptivas de satisfacción/ insatisfacción laboral, c) unión de fragmentos textuales concordantes con cada variable descriptiva, d) descripción analítico-reflexiva del contenido de cada variable (identificando, en este caso, miradas compartidas entre los entrevistados) y e) determinación de la frecuencia de cada descriptor, asignando una puntuación de 1 ó 0 a cada individuo entrevistado dependiendo de si la variable lo representaba o no (Onwuegbuzei y Leech, 2007).

\section{RESULTADOS Y DISCUSIÓN}

A la pregunta de sí en términos generales se sienten satisfechos con sus trabajos, el 75\% de los entrevistados respondió afirmativamente, usando expresiones cargadas de calificativos positivos: "bastante (Entrevistados 11 y 22)", "por su puesto (Entrevistados 9 y 14)", "me gusta mucho (Entrevistados 2, 7 y 17)", "sin duda (Entrevistado 4)". Esto significa que, desde un enfoque unidimensional (que valora y evalúa la experiencia como un todo), los extensionistas que hicieron parte del estudio tiene una actitud positiva hacia el mismo y describen globalmente su experiencia como favorable, a pesar de reconocer y mencionar la existencia de algunos aspectos o circunstancias negativas.

Desde el enfoque multidimensional, afirmando que una actitud genérica resulta del cruce de actitudes específicas en relación con diferentes aspectos del trabajo, se encontró que, al evaluarse separadamente, algunos aspectos específicos son negativamente valorados, sin 
detrimento de la apreciación positiva concedida a la experiencia laboral en términos globales. A continuación se presentan las valoraciones dadas por los extensionistas a factores problemáticos constitutivos, identificados a partir del análisis de sus propias narrativas (Figura 1). Se optó por presentar los factores de mayor puntuación (los más repetidos o de mayor frecuencia), sin separarlos formalmente entre satisfactores e insatisfactores, considerando la simultaneidad y relación (Segurado y Agullo, 2002).

\section{Gusto por la profesión ejercida}

Para el $83 \%$ de los entrevistados, ser extensionista es fuente de complacencia y autorrealización: "Mi trabajo es algo que me complace enormemente, es algo que me define como profesional y persona. Cuando me preguntan qué soy o quién soy, yo siempre me presento como extensionista porque eso es lo que soy (Entrevistado 9)", "me siento plenamente identificado con lo que hago, fue el camino profesional que yo escogí, trabajar por la gente y ayudar a la gente del campo (Entrevistado 12).

Analíticamente, la valoración positiva otorgada al gusto por el oficio realizado es reflejo de auto-concebir la extensión, más que como un simple trabajo, como una actividad integrada a un proyecto personal de vida, consonante con lo que el sujeto quiere ser y hacer. Sobre esta lectura, para los entrevistados ejercer el oficio de extensionista es un medio que contribuye a materializar parte de ese proyecto individual de vida, desplegado en un contexto y tipo de sociedad determinada que ofrece las condiciones para ello: el mundo rural local y regional.

En concreto, el gusto por el oficio está ligado a la posibilidad que este brinda de definir lo que para ellos significa y permite ser extensionista rural: "realizar un trabajo que me llena como persona (Entrevistado 3)", "contribuir a construir un mundo más equitativo (Entrevistado 13)", "dar sentido a la propia existencia (Entrevistados 1 y 9)" y "ayudar a generar condiciones de bienestar social entre los menos favorecidos (Entrevistados 14 y 21)".
En relación con los antecedentes personales, lo experimentado y aprendido en las instituciones en las que se formaron (facultades de agronomía, ciencias agropecuarias, trabajo social e áreas afines) en parte motivó su inclinación vocacional. La aproximación teórica y práctica a las sociedades rurales, la historia agraria, los sistemas de extensión y el desarrollo rural influenció la proyección personal y social por "el trabajo con y por la gente del campo (Entrevistados 7, 11 y 14)", hecho que refuerza los intereses hacia el trabajo social comunitario.

En referencia al ámbito local, trabajar en una región en la que predominan los pequeños productores de café, actores hoy más que nunca empobrecidos y socialmente vulnerables debido a las situaciones ya referenciadas, ofrece las condiciones para que "trabajar con los más pobres del campo (Entrevistados 9, 13 y 17)" se concrete. Se trata de un contexto sociocultural específico (un sistema de normas, valores, instituciones y esferas de actividad social) en el que los extensionistas pueden dar vía libre a sus pretensiones de proyección social y personal, asumiendo compromisos y responsabilidades desde su quehacer profesional, lo cual es motivo de satisfacción y bienestar.

\section{Posibilidad de proyección social}

Para el 78\% de los entrevistados, la proyección social, entendida como la posibilidad de promover impactos positivos sobre la vida de otras personas y el entorno físico-social rural más inmediato, es motivo de satisfacción:

"Lo que más me enorgullece es saber que con mi trabajo estoy ayudando a resolver problemas, a resolver problemas de la gente. Así mi contribución sea pequeñita, yo soy parte de la solución y no sólo un observador pasivo (Entrevistado 7)".

En este caso, intervenir sobre problemas y contribuir a superar ciertas necesidades de los individuos y las comunidades con los que mantienen contacto es una acción grata, deseada y valorada. Según lo expuesto por

Tabla 1. Ejes de indagación y preguntas orientadoras del diálogo

\begin{tabular}{ll}
\hline Ejes de indagación & Preguntas guía \\
\hline Situaciones promotoras de complacencia e insatisfacción laboral & $\begin{array}{l}\text { ¿Qué hechos o situaciones de la cotidianidad laboral les suscita } \\
\text { emociones placenteras?, ¿cuáles le disgustan o generan } \\
\text { sentimientos de rechazo o poco gratos? }\end{array}$ \\
\hline $\begin{array}{l}\text { Opiniones evaluativas y valorativas sobre cada situación } \\
\text { problemática señalada }\end{array}$ & $\begin{array}{l}\text { ¿Qué opinión evaluativa les merece cada una de estas } \\
\text { circunstancias?, ¿cómo las juzgan? }\end{array}$ \\
\hline $\begin{array}{l}\text { Soporte empírico de cada juzgamiento } \\
\text { Particularidades de los contextos microsociales y macrosociales } \\
\text { en los que los extensionistas desarrollan su práctica }\end{array}$ & $\begin{array}{l}\text { ¿Qué elementos del entorno físico y situacional (bien sea de índole } \\
\text { cultural, socioeconómica, institucional o cualquier otra) subyacen } \\
\text { tras cada valoración evaluativa? }\end{array}$ \\
\hline
\end{tabular}

Rev. Ceres, Viçosa, v. 62, n.3, p. 241-250, mai-jun, 2015 
los entrevistados, dicha práctica debería ser asumida como básica e ineludible en el quehacer de todo extensionista: "para eso estamos aquí, eso es lo que venimos a hacer. Poder ayudar a la gente es lo que debería animarnos a hacer bien nuestro trabajo (Entrevistado 20)".

De acuerdo con lo encontrado, la complacencia producida por la posibilidad de proyectarse, además de sustentarse en la satisfacción generada por el compromiso con causas altruistas, está específicamente ligada al interés por materializar ciertos valores considerados positivos, y hoy casi dados por perdidos en los procesos de interacción social (solidaridad, fraternidad, compromiso, altruismo, entre los más mencionados), asumiendo su puesta en práctica como mecanismo de distinción e identidad personal.

Esta distinción se basaría en actuar en contra de algunos contravalores considerados negativos cuando son vistos bajo un esquema comparativo. Confrontar indiferencia a responsabilidad social, integracionismo a individualismo, economicismo a solidaridad, entre los más inferido en las narrativas de los entrevistados, son ejemplos de ello. Son valores adoptados como orientadores de la práctica profesional, resaltados a diferencia de otros profesionales:

"Yo no sólo actúo en función del dinero, como muchos agrónomos dedicados a la venta a comisión de agroquímicos. Sin que esto quiera decir que no viva de mi trabajo, yo sí trato de actuar en beneficio de la gente, haciendo realidad mi decisión de ayudar a mis campesinos. Si no fuera así, hace rato estuviera en otro lado (Entrevistado 14)".

En este contexto, aunque la función misional de las unidades de extensión direcciona a este comportamiento: "prestar asistencia técnica a los campesinos y pequeños productores, con el fin de mejorar los sistemas de producción, el nivel de ingresos y la calidad de vida”, propósito misional típico de las UMATA integradas al estudio, es más la convicción de cada extensionista la que promueve estos valores. En este caso, sus convencimientos éticos y políticos, bien sean individuales o compartidos con otros miembros de los equipos de trabajo, son los que orientan sus conductas.

\section{Alcance y visualización de logros}

Para el 74\% de los entrevistados, alcanzar metas y visualizar sus resultados, principalmente cuando estos perduran en el mediano y largo plazo, generan emociones y sentimientos complacientes: "Para mí es motivo de orgullo y alegría lograr finalizar con éxito un proyecto, sobre todo cuando sé que éste puede seguir andando solo, manejado totalmente por la comunidad (Entrevistado 23)".

En este caso, la satisfacción se deriva del sentimiento que produce alcanzar satisfactoriamente los objetivos a cumplir, sabiendo que su intervención en las comunidades tuvo un efecto permanente. Apreciar la independencia de las comunidades, vista en la capacidad desarrollada para dar continuidad a los procesos inicialmente impulsados por los extensionistas, produce sentimientos de autorrealización y autoestima, a su vez generadores de complacencia con el trabajo: "me siento orgulloso de que así se den las cosas, de que mi contribución haya sido efectiva (Entrevistado 3).

Sumado a lo anterior, la investigación reveló que dicho sentimiento está con fuerza asociado al temor a fallar en las tareas encomendadas (disminuir el riesgo de inseguridad alimentaria, fomentar y conseguir que la comunidad se organice alrededor de la producción y la comercialización agrícola, entre lo más mencionado).

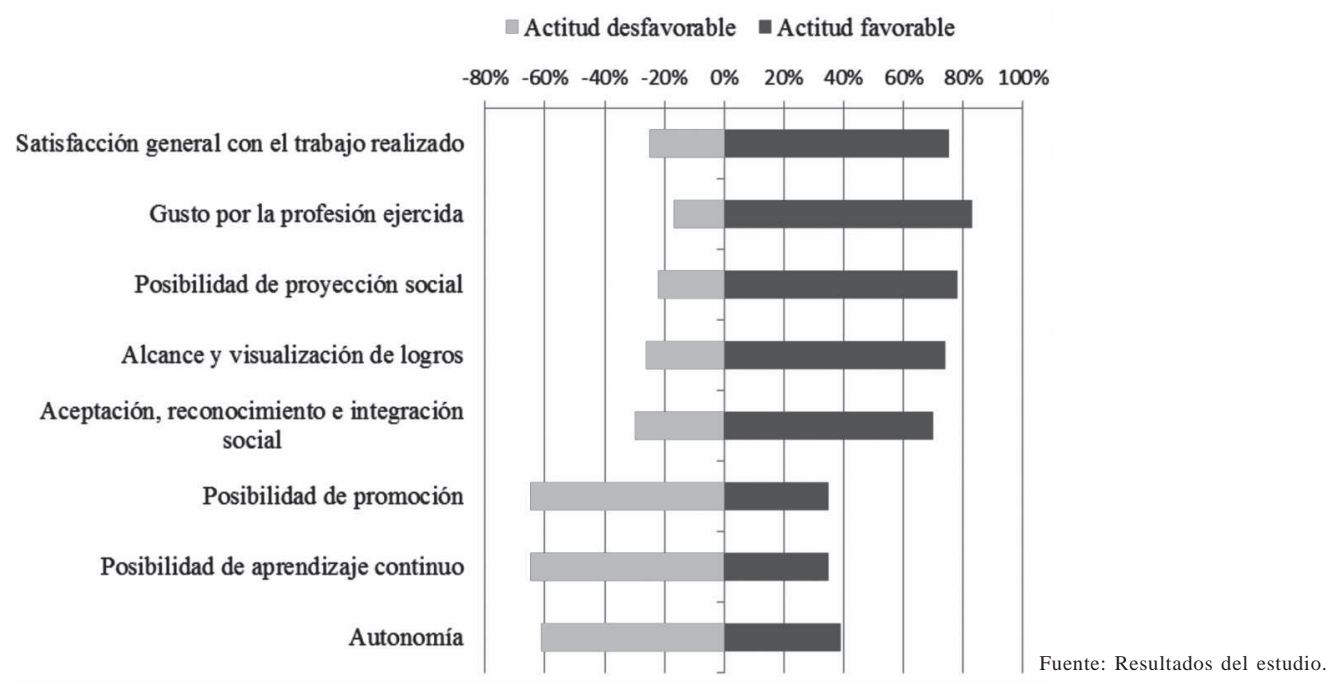

Figura 1. Valoración actitudinal de factores de satisfacción/insatisfacción con el empleo de extensionista rural 
Esto ocurre teniendo en cuenta que, en el sitio de estudio, la práctica profesional se desarrolla en un escenario de desconfianza, en el que los productores o usuarios de sus servicios medianamente confían en el éxito de los programas o proyectos propuestos, debido a experiencias previas fallidas y a la falta de continuidad de los mismos. Superar las dificultades, culminando con éxito lo planteado y recuperando la confianza de las comunidades, es otra fuente de satisfacción, en este caso.

En lo concerniente al mundo laboral, la evaluación de desempeño a partir de indicadores y metas a cumplir es algo que causa tensión y ansiedad en los extensionistas. En este caso, el número de actividades realizadas, valorada con los mínimos o registros institucionalmente impuestos, define cuándo o no sé ha alcanzado un objetivo, meta traducida en algún indicador dentro de los sistemas de control de gestión:

"Para mí, cumplir con la meta establecida, capacitar a 185 productores en BPA (Buenas Prácticas Agrícolas), por ejemplo, de acuerdo con el Plan de Acción, me llena de satisfacción en la medida que siento que he cumplido con lo tarea impuesta. Eso sí, muchas veces yo soy consciente de que debo sacrificar calidad por cantidad, lo cual llega a causarme un poco de inconformidad conmigo mismo y también vergüenza, a veces, con los productores (Entrevistado 22)".

En estas circunstancias, cumplir con las metas formuladas desde la lógica institucional (hacer lo que más se pueda durante el periodo lectivo de un alcalde o presidente municipal, demostrándolo a partir de indicadores de gestión que impresionen al electorado) es tomado por los propios extensionistas como parámetro de autoevaluación y auto-juzgamiento. Alcanzar esas metas numéricas, con frecuencia excesivas para las posibilidades y recursos disponibles, asumiendo además el riesgo de que el trabajo realizado sea de mala calidad (Landini, 2012), genera parcialmente en los extensionistas actitudes positivas.

A propósito de esta parcialidad, la satisfacción generada por cumplir con lo solicitado, hecho que supone seguir a cabalidad los mandatos institucionales establecidos sobre qué hacer, cuánto hacer y cómo hacerlo, llega a chocar con la insatisfacción producida por "no haber hecho lo que en verdad se hubiera tenido que hacer (Entrevistado 11)". En este caso, el sentimiento de culpa causado por no haber decidido en su momento trasgredir los lineamientos impuestos produce tensión e inconformidad, en un contexto que, próximo a lo expuesto por Landini (2013) para el caso uruguayo, predominan sistemas de planificación centralizados que impone rubros específicos que no siempre corresponden con las necesidades o prioridades de las poblaciones o localidades intervenidas. Este hecho revela la presencia de sentimientos encontrados (me siento bien por un lado, pero mal por otro) que tornan confuso o inestable el estado emocional de los sujetos en relación con el alcance y la visualización de logros.

\section{Aceptación, reconocimiento e integración social}

Para el $70 \%$ de los entrevistados, sentirse acogidos por aquellos con quienes cotidianamente se relacionan durante el ejercicio profesional es un elemento de gratificación y complacencia:

"Lo que más disfruto de mi trabajo es la oportunidad de relacionarme a diario con la gente sencilla del campo, con gente que me aprecia y valora mi trabajo, así hayamos, sin querer, cometiendo errores. Llegar a sus casas, ser recibidos con un tinto [café], sentarme con ellos un rato a comentar no sólo sobre la producción de la finca, sino sobre cómo va la familia, la situación del país, la forma como ellos ven los problemas del campo e, incluso, para recibir críticas constructivas sobre la forma cómo funciona la UMATA, para mí es totalmente grato. El agradecimiento, el aprecio, la confianza, el buen recibimiento, la familiaridad con que me tratan es lo que más me agrada cada vez que hago una visita. (Entrevistado 13)".

En primer lugar, la valoración positiva de este factor se sustenta en la satisfacción producida por ser aceptados y queridos por aquellos que, en palabras de varios de los entrevistados, justifican su existencia como profesionales del agro: los productores y otros habitantes del campo a quienes acompañan y prestan sus servicios. En este caso, la proximidad no sólo profesional sino también afectiva con los otros, la aceptación como parte de su círculo social y la estabilidad de estas relaciones soportan los sentimientos de aprecio, reconocimiento y acogida en conjunto generadores de actitudes favorables mediadas por lo emocional y afectivo.

Se trata de sentimientos no siempre experimentados en otros sub-ámbitos del mismo universo microsocial de trabajo, en donde, a pesar de la existencia de camaradería y relaciones armónicas entre colegas, el reconocimiento y el aporte de la labor realizada es minimizado:

"Aquí en la alcaldía [municipal] nosotros somos más o menos cenicientas. Aunque no lo digan abiertamente, los otros nos ven siempre como los de menos estatus, los que trabajamos con los campesinos y la gente pobre del campo, con la gente que casi a nadie le importa, para la que hay menos presupuesto de inversión, con la gente que, como ellos muchas veces creen, el gobierno tiene que mantener para que sobreviva. A mí esto realmente no me importa, pero sí me gustaría que la visión fuera distinta. (Entrevistado 21)". 
Analíticamente, el bajo reconocimiento otorgado a los miembros de las UMATA en comparación con el concedido a los profesionales de otras dependencias (secretarías de planeación, salud y educación, entre las más mencionadas) es suplido por el recibido de los campesinos, pequeños productores y otros actores locales con los que interactúan durante su labor (pares extensionistas, líderes comunitarios, rectores y docentes de colegios agropecuarios, entre los destacados). Se trata de un sentimiento positivo asumible como mecanismo de compensación emocional y afectiva, que impulsa el acercamiento a los otros, la procura, la conservación y el disfrute de dicha interacción social, lo cual a su vez fomenta y refuerza el compromiso con la búsqueda del bienestar de esos otros.

En relación con el contexto social local, el bajo estatus otorgado tendría influencia en la lectura realizada acerca del espacio físico-social en el que los extensionistas laboran. Por asociación, la visión dicotómica entre lo urbano y lo rural predominante en el escenario local, mirada que principalmente discrimina entre lo moderno y lo atrasado, es proyectada sobre los extensionistas. "Trabajar en el campo y con los pobres del campo" los ubica en la base de una escala jerárquica subjetivamente construida, hecho que da cuenta de la segregación aún existente tanto en la división como en la espacialización social del trabajo.

\section{Posibilidades de promoción}

Para el $65 \%$ de los entrevistados, la mínima posibilidad de promoción interna, entendida como el ascenso a un cargo superior con un mayor salario y más responsabilidades, genera emociones y valoraciones negativas:

“Aunque puede estar muy a gusto aquí, aunque estoy haciendo lo que me gusta, se que laboralmente estoy estancado. Ascender, aumentar mi salario por encima del reajuste anual de ley y que me sea reconocida mi experiencia, difícilmente se dará. Sé que esto también lo sienten otros, como algunos que hoy están buscando otras posibilidades de trabajo, como ingresar al magisterio, a la secretaría [departamental] de agricultura o a instituciones como la policía nacional (Entrevistado 4)".

En la práctica, esta situación deriva de la inexistencia en las unidades de extensión de planes de carrera o sucesión de cargos a los que puedan aspirar, circunstancia que negativamente interfiere sobre los planes de proyección profesional. En situaciones ideales, los ascensos tendría que responder al reconocimiento de la valía, los conocimientos, la antigüedad, el liderazgo y los méritos demostrados, factores a los que en los sitios de estudio se les otorga poco o ningún valor: "Vetera- nos, jóvenes y practicantes resultamos todos haciendo lo mismo, sin tener en cuenta la experiencia o los logros que cada uno haya alcanzado, diferenciándose en casi nada los salarios recibidos (Entrevistado 18)".

Como ilustra el anterior testimonio, en el contexto de estudio, el grado de satisfacción está mediado por el cruce entre remuneración y promoción, obtenido del contraste entre el salario propio y el recibido por colegas de formación similar, pero con menos experiencia o antigüedad en la institución. En este caso, la nivelación en un sistema de valoración salarial (sistema que sin ser injusto o inequitativo reconozca la trayectoria profesional de cada quien), en términos generales, soporta la inconformidad.

De los testimonios también es posible deducir que uno de los resultados de la mínima posibilidad de ascenso es la fuga de talentos. Para el $48 \%$ de los entrevistados, grupo mayoritariamente integrado por profesionales de recién egreso y menos de cuatro años de vinculación, "hacer carrera en la UMATA (Entrevistado 11)" no es atractivo ni considerado en los planes a mediano y largo plazo, debido nuevamente al sentimiento de estancación. La "necesidad de progresar laboral y profesionalmente (Entrevista No. 18)" conlleva al retiro de las unidades de extensión y a la vinculación a otras organizaciones afines al desarrollo rural, cuando no ajenas a éste, lo que da cuenta de las consecuencias extremas derivadas de la insatisfacción con el factor promocional. Sobre el tema, estudios similares han mostrado que mayores oportunidades de desarrollo profesional están directamente relacionadas con mayores niveles de satisfacción laboral, un alto compromiso con la organización vinculante y una mínima intención de cambio de trabajo (Arribas, 2007; Acker, 2014), situación comprobada por este estudio, cuando se lee en sentido inverso.

En relación con el contexto organizacional, el tamaño de las unidades de extensión, el bajo monto de los recursos de operación disponibles, sumado a la simplicidad del organigrama interno, dificulta contar con estrategias de promoción profesional, lo cual restringe las aspiraciones personales, reafirmando sentimientos de insatisfacción con el trabajo.

Concretamente, en unidades básicamente compuestas por un director (para el caso de estudio en su totalidad nombrados en respuesta a su filiación política) y un número limitado de asistentes técnicos (cinco en promedio, en este caso), para cuyo funcionamiento el municipio o la entidad correspondiente destina un presupuesto bajo (del 1 al 3\% del general), establecer planes de carrera, que incluyan modalidades de ascenso y movimientos ascendentes en la escala salarial, resulta organizativamente poco viable. 


\section{Posibilidades de aprendizaje continuo}

Para el $65 \%$ de los entrevistados, el mínimo impulso al aprendizaje continuo es un factor de descontento laboral. "La poca atención dada a la necesidad de estar actualizados (Entrevistado 14)", "la casi nula preocupación por mejorar el perfil de los funcionarios de la UMATA", "el bajo compromiso de la administración con la formación del capital humano (Entrevistado 9)", entre otras apreciaciones, describen la esencia del problema.

De acuerdo con lo encontrado, al sentimiento de inconformidad asociado al bajo apoyo institucional para la formación como vía de promoción profesional, se suma la sensación derivada de sentirse cada vez menos preparados para enfrentar una realidad agrícola y rural cambiante. En síntesis, este sentimiento resulta de reconocerse como mínimamente calificados para: a) asumir tareas para las cuales no han sido directamente formados (labores relacionadas con la gestión ambiental local, incluyendo la mediación en la resolución de conflictos por del recurso agua, entre lo más comentado), b) superar la desactualización de conocimiento, y c) actuar en situaciones que demandan capacidades inter y transdisciplinarias, que superan la especialidad profesional (tomar decisiones no sólo fundamentadas en criterios técnicos o técnico-económicos sino también socioambientales, políticos y culturales, principalmente).

Con relación al ámbito institucional, la carencia de un sistema de beneficios e incentivos que contemplen la formación profesional, esta vez asumido como medio para asegurar el mejoramiento permanente de las unidades de extensión, nutre el sentimiento de insatisfacción laboral. En este caso, proporcionar a los funcionarios herramientas que les permita mantenerse hábiles en su oficio no es institucionalmente tomado como forma de agregar valor a las UMATA. En términos generales, este hecho se expresa en la reducción gradual de la eficacia y calidad de los servicios ofrecidos (apreciación recurrente entre los mismos profesionales), el deterioro de la imagen institucional (pérdida de credibilidad por parte de los usuarios) y la percepción acerca de la idoneidad de los extensionista, lo cual individual y colectivamente genera insatisfacción con el trabajo.

\section{Autonomía}

Para el $61 \%$ de los entrevistados, los bajos niveles de autonomía, entendida como la posibilidad de tomar decisiones propias concernientes al quehacer profesional cotidiano, generan sentimientos de inconformidad y frustración:

"Para mí no deja de ser difícil tener que hacer al pie de la letra lo que un director o un asesor diga, sobre todo cuando yo estoy casi seguro de que las cosas podrían planearse de otra forma, que con otras actividades y proyectos se podrían tener mejores resultados o lograr mayor impacto sobre las comunidades. Yo, sin ser el único, creo que tengo mucho que aportar. Mi conocimiento directo de las zonas rurales, de su gente y de sus problemáticas me lo permiten. Sin embargo, yo no tengo poder de decisión, por lo que casi siempre me tengo que quedar haciendo sólo lo que hay que hacer (Entrevistado 3)".

En este caso, siendo conscientes de que en las UMATA las decisiones son tomadas por una persona ubicada en la cima de la pirámide organizacional (el director o el mismo alcalde), más que en la indisposición con el orden jerárquico, la insatisfacción se soporta en un elemento emocional: la frustración producida por las pocas posibilidades de auto-reforzar los sentimientos de eficacia personal, creatividad y competencia, mediante la propuesta y aplicación de soluciones ideadas a problemas surgidos en la práctica profesional (seguir una estrategia novedosa de apoyo a la gestión comunitaria, usar otros criterios sustentados para la selección de los beneficiarios de un proyecto, prioriza actividades en razón de los conocimientos a profundidad de las necesidades apremiantes, entre lo destacado).

En este contexto, poder decidir qué hacer o cómo hacer una tarea, es decir, contar con cierta autonomía personal en el trabajo, es el elemento faltante. En similar sentido, ser tratados como ejecutores de tareas puntuales, más que como agentes capacitados para proponer soluciones y tomar decisiones, refuerza la insatisfacción aludida.

En el ámbito institucional, las oportunidades de contar con más grados de libertad, independencia y discreción en el actuar son limitados por la inflexibilidad de algunas directrices definidas por instancias de mayor escala jerárquica (el Ministerio de Agricultura y Desarrollo Rural, el Instituto Colombiano Agropecuario, principalmente), en donde se precisa qué hacer y cómo hacer las cosas. Se trata de instrucciones que restringen el proceder autónomo los gobiernos locales, y se extienden a la práctica de los extensionistas rurales, con la consecuente generación de insatisfacción.

\section{Inestabilidad laboral}

Entre el $63 \%$ de los entrevistados, la inestabilidad laboral, en este caso asociada a la prevalencia de contratos de corta duración (de 9 a 10 meses al año quedando tres o dos cesantes), genera sentimientos de inseguridad:

"Cada año es prácticamente lo mismo, llega el fin de año y no sabemos si vamos a seguir, si nos van a volver a contratar en febrero, si van a contratar a otros o si va a haber suficiente presupuesto para dejarnos a todos. Por lo menos para mí, que tengo constituida una familia, la situación siempre es difícil (Entrevistado 19)". 
En este caso, el sentimiento de incertidumbre se sustenta en la tensión que afrontan al trabajar en un ambiente que no les garantiza la permanencia en el cargo ni la estabilidad de un ingreso, ya que la contratación se realiza por periodos cortos con interrupción periódica.

Esta situación, más allá de influenciar simplemente lo contractual, en coincidencia con lo expuesto por Abrajan, Contreras y Montoya (2009), repercute sobre la vida social e individual, con efectos sobre el bienestar personal y familiar: restricciones en el acceso a bienes materiales y servicios, aplazamiento de la toma de ciertas decisiones (constituir un hogar propio, conseguir independencia familiar, sobre todo para el caso de los más jóvenes), no poder ahorrar y saber que va a ser difícil conseguir otro empleo, circunstancias que generan sentimientos de escepticismo y miedo.

En relación con el ámbito institucional, a las formas actuales de contratación y flexibilización laboral (contratos de trabajo atípicos, en este caso de corta duración, que no prevén protección frente al despido, principalmente), se suma la asignación clientelar de plazas de trabajo. En las unidades de extensión que hicieron parte del estudio, adjudicar puestos a personas partidistamente afines a los gobernantes o directivos de turno suele ser común, actuación arbitraria y politizada que, como también observó Landini (2012), genera un ambiente de inequidad en el que no son los méritos de cada quien los que garantizan beneficios laborales, sino otros de dudosa índole.

Dar prioridad de vinculación y permanencia a individuos políticamente más rentables (aquellos que brindan mayor rendimiento para atraer apoyo y mantener relaciones políticas) sustenta esta acción que omite el reconocimiento al mérito profesional y personal como medida transparente de ascenso y continuidad en el trabajo, hecho que acentúa la incertidumbre causante de inconformidad.

En términos generales, se trata en su conjunto de factores de satisfacción e insatisfacción laboral que al hacer parte de un todo consiguen bien sea equilibrar o desequilibrar en sentido opuesto el efecto de uno sobre otro, produciendo, para el caso de estudio, un sentimiento general de satisfacción con el trabajo, a pesar de la presencia de situaciones problemáticas. En este sentido, el gusto por el oficio de extensionista equilibra la insatisfacción originada por las mínimas posibilidades de promoción laboral. De manera similar, el mínimo estímulo a la formación continuada podría ser compensado por el aprendizaje continuo resultante de la interacción directa y sostenida con las gentes del campo, mecanismo que permite compensar la afección de un factor con el de otro.

\section{CONCLUSIONES}

Se concluye que la mayoría de los extensionistas entrevistados muestra una actitud positiva hacia el trabajo y describe globalmente su experiencia como satisfactoria, aun reconociendo y señalando la existencia de algunos aspectos negativos. Para ellos, los factores más significativos de satisfacción/ insatisfacción son: a) gusto por la profesión ejercida, b) posibilidad de proyección social, c) alcance y visualización de logros, d) aceptación, reconocimiento e integración social, e) posibilidad de promoción, f) posibilidad de aprendizaje continuo, g) autonomía y h) estabilidad laboral.

Considerando que la práctica profesional no acontece en el vacío sino en un contexto social específico, se concluye que las actitudes en relación con los factores señalados están principalmente influenciadas por: a) la condición de vulnerabilidad socioeconómica afrontada por los pequeños productores de café, en un contexto en que ayudar a estos actores, además de exaltar el gusto por el trabajo, es una oportunidad para concretar el anhelo de proyección social, b) la inflexibilidad de las políticas y directrices sectoriales, que limitan la autonomía y la creatividad en la toma de decisiones, c) las relaciones partidistas-clientelistas predominantes al interior de la administración municipal, que restan valor a la meritocracia y favorece la inestabilidad laboral, d) el bajo presupuesto y la estructura organizativa de las unidades locales de extensión, que conjuntamente limitan la posibilidad de establecer un sistema de incentivos acorde con la labor realizada, y e) la predominancia de modalidades atípicas de contratación laboral, que incrementan la desprotección en relación con la seguridad del empleo y la seguridad social, principalmente.

Por otro lado, considerando que los sentimientos positivos o negativos frente a diferentes situaciones de trabajo son un reflejo del contexto laborar (sano o viciado) en el que los extensionistas a diario se desenvuelven, se prevé que interesarse en sus actitudes y asumirlas como guías para avanzar hacia un ambiente de trabajo más armónico sería una decisión en pro de un mejor servicio de extensión. Se asume que un extensionista satisfecho traslada su satisfacción a la vida personal y laboral, sentimiento que a su vez redunda en el bienestar de las comunidades rurales que acompañan. En este orden, desconocer o hacer caso omiso de dichos sentimientos eleva el riesgo de crear o reproducir escenarios no favorables que limiten el compromiso con la labor social realizada, o bien la reafirmación continúa de lo que podría reconocerse como el espíritu o la vocación del extensionista. 
Como consideración general se presume que optimizar el funcionamiento de las unidades de extensión, y la efectividad de los proyectos y programas orientados a elevar los niveles de bienestar social rural, además de demandar: a) incrementar las partidas presupuestales disponibles, b) adoptar modelos de gestión acordes con las particularidades del entorno, c) favorecer la articulación entre las distintas instituciones participantes en procesos desarrollo rural, d) establecer vínculos estrechos y en doble vía con los centros de investigación, e) mejorar el acceso a redes de información, f) garantizar la continuidad y la estabilidad del servicio, g) conocer a profundidad las zonas de trabajo y las dinámicas sociales que allí tienen lugar, también exige, como aquí fue presentado, h) mejorar los grados de satisfacción laboral de los extensionistas rurales.

Por último es preciso tener en cuenta que los factores de satisfacción laboral no responden a situaciones estables o genéricas, lo cual implica tener siempre presente que la vivencia laboral de los extensionistas rurales no puede plantearse con un único matiz, desconociendo la especificidad de los innúmeros contextos de ocurrencia, escenarios limitados en el tiempo y el espacio. En esta vía, los resultados de este estudio microsocial localizado puede ser asumido como insumo para estudios comparativos a futuro.

\section{REFERENCIAS}

Abrajan M, Contreras J \& Montoya S (2009) Grado de satisfacción laboral y condiciones de trabajo: una exploración cualitativa. Enseñanza e Investigación en Psicología, 14:105-118.

Acker G (2004) The effect of organizational conditions (role conflict, role ambiguity, opportunities for professional development, and social support) on job satisfaction and intention to leave among social workers in mental health care. Community Mental Health Journal, 40:65-73.

Arribas A (2007) El equilibrio personal y profesional como valor de la cultura corporativa. Organicom, 4:49-63.

Barón J (2010) Geografía, café y prosperidad en los Andes Occidentales de Colombia. Revista de Economía del Rosario, 13:117-190.

Cano C, Vallejo C, Caicedo E, Amador J \& Tique E (2012) El mercado mundial del café y su impacto en Colombia. Bogotá, Banco de la República. 56p.

Gamero C (2005) Análisis microeconómico de la satisfacción laboral. Madrid, Consejo Económico y Social. 273p.

Hesse-Biber S \& Leavy P (2010). The practice of qualitative research. $2^{\text {a }}$ ed. Los Angeles, Sage. 400p.

Hontagas P \& Peiró J (2002) Ajuste persona-trabajo. In: Peiró J \& Prieto F (Eds.) Tratado de Psicología del Trabajo: Vol I: La actividad laboral en su contexto. Madrid, Síntesis. p. 251-282.

Jodelet D (2006) Place de l'expérience vécue dans les processus de formation des représentations sociales. In: Haas V (Ed.) Les savoirs du quotidien. Rennes, PUR. p. 235-255.

Kvale S (1983) The qualitative research interview: A phenomenological and a hermeneutical mode of understanding. Journal of Phenomenological Psychology, 14:171-196
Kvale S \& Brinkmann S (2009) Interviews. Learning the craft of qualitative research interviewing. $2^{\mathrm{a}}$ ed. Los Angeles, Sage. 352p.

Landini F (2012) Problemas de la extensión paraguaya: modelos de extensión en la encrucijada. Cuadernos de Desarrollo Rural, 9:127149.

Landini F (2013) Necesidades de los extensionistas rurales paraguayos desde la perspectiva de su función, sus problemas y sus intereses. Trabajo y Sociedad 20:149-160.

Landy F \& Conte J (2005) Psicología industrial: Introducción a la psicología industrial y organizacional. $6^{a}$ ed. México, McGraw-Hill. $630 \mathrm{p}$.

López F \& Castro J (2010) Redimensionamiento de la extensión agrícola como práctica educativa comunitaria ante los embates neoliberales. Revista Científica UDO Agrio, 10: 88-93.

Méndez M (2006) Los retos de la extensión ante una nueva y cambiante noción de lo rural. Revista Facultad Nacional de Agronomía, 59:34073423.

Morin E, Tonelli M \& Vieira A (2007) O trabalho e seus sentidos. Psicologia \& Sociedade, 9:47-56.

Morse J (1995) The significance of saturation. Qualitative Health Research, 5:147-149.

Nates B \& Velásquez P (2009) Territorios en mutación. Crisis cafetera, crisis del café. Cuadernos de Desarrollo Rural, 6:11-33.

Onwuegbuzei A \& Leech N (2007) A call for qualitative power analyses. Quality and Quantity. 41:105-121.

Robbins S \& Coulter M (2005) Administración. México, Pearson Education. 640p.

Rodríguez L, La OM, Fonseca M, Guevara F, Hernández A \& Jiménez M (2009) Extensionismo o innovación como proceso de aprendizaje social y colectivo. ¿Dónde está el dilema? Revista Cubana de Ciencia Agrícola, 43:387-394.

Rossi V (2011) Aportes metodológicos para el asesoramiento técnico y la extensión rural. Revista Cangüe, 31:51-60.

Segurado A \& Agulló E (2002) Calidad de vida laboral: hacia un enfoque integrador desde la psicología social. Psicothema, 14:828-836.

Sheth J, Mittal B \& Newman B (2001) Comportamento do cliente: indo além do comportamento do consumidor. São Paulo, Atlas. 800p.

Spector P (2012) Industrial and organizational Psychology. 6 ${ }^{\mathrm{a}}$ ed. Estados Unidos, John Wiley \& Sons. 441p.

Rev. Ceres, Viçosa, v. 62, n.3, p. 241-250, mai-jun, 2015 